\title{
A MORE FAULT TOLERANT APPROACH TO ADDRESS RESOLUTION FOR A MULTI-LAN SYSTEM OF ETHERNETS
}

\section{STATUS OF THIS MEMO}

This memo discusses an extension to a Bridge Protocol to detect and disclose changes in neighbouring host address parameters in a MultiLAN system of Ethernets. The problem is one which is appearing more and more regularly as the interconnected systems grow larger on Campuses and in Commercial Institutions. This RFC suggests a protocol enhancement for the Internet community, and requests discussion and suggestions for improvements. Distribution of this memo is unlimited.

\section{ABSTRACT}

Executing a protocol $\mathrm{P}$, a sending host $\mathrm{S}$ decides, through $\mathrm{P}^{\prime} \mathrm{S}$ routing mechanism, that it wants to transmit to a target host $\mathrm{T}$ located somewhere on a connected piece of $10 \mathrm{Mbit}$ Ethernet cable which conforms to IEEE 802.3. To actually transmit the Ethernet packet, a 48 bit Ethernet/hardware address must be generated. The addresses assigned to hosts within protocol $\mathrm{P}$ are not always compatible with the corresponding Ethernet address (being different address space byte orderings or values). A protocol is presented which allows dynamic distribution of the information required to build tables that translate a host's address in protocol $\mathrm{P}^{\prime} \mathrm{s}$ address space into a 48 bit Ethernet address. An extension is incorporated to allow such a protocol to be flexible enough to exist in a Transparent Bridge, or generic Host. The capability of the Bridge to detect host reboot conditions in a multi-LAN environment is also discussed, emphasising particularly the effect on channel bandwidth. To illustrate the operation of the protocol mechanisms, the Internet Protocol (IP) is used as a benchmark [6], [8]. Part 1 presents an introduction to Address Resolution, whilst Part 2 discusses a reboot detection process.

DEFINITIONS :

CATENET: a group of IP networks linked together IP : Internet Protocol 
PART 1

\section{INTRODUCTION}

In the Ethernet, while all packets are broadcast, the hardware interface selects only those with either the explicit hardware broadcast address or the individual hardware address of this interface. Packets which do not have one of these two addresses are rejected by the interface and do not get passed to the host software. This saves a great deal of otherwise wasted effort by the host software having to examine packets and reject them. If the interface hardware selected packets to pass to the host software by means of the protocol address, there would be no need for any translation from protocol to Ethernet address. Although it is very important to minimize the number of packets which each host must examine, so reducing especially needless inspections, use of the hardware broadcast address should be confined to those situations where it is uniquely beneficial. Perhaps if one were designing a new local network one could eliminate the need for an address translation, but in the real world of existing networks it fills a very important purpose. A rare use of the broadcast hardware address, which avoids putting any processing load on the other hosts of the Ethernet, is where hosts obtain the information they need to use the specific and individual hardware addresses to exchange most of their packets.

\section{REASONING BEHIND ADDRESS RESOLUTION}

The process of converting from the logical host address to the physical Ethernet address has been termed ADDRESS RESOLUTION, and has prompted research into a method which can be easily interfaced, whilst at the same time remaining portable.

The Ethernet requires 48 bit addresses on the physical cable [11] due to the fact that the manufacturers of the LAN interface controllers assign a unique 48 bit address during production. Of course, Network Managers do not want to be bothered using this address to identify the destination at the higher-level. Rather, they would prefer to assign their logical names to the hosts within their supervision, and allow some lower level protocol to perform a resolving operation. Most of these logical protocol addresses are not 48 bits long, nor do they necessarily have any relationship to the 48 bit address space.

For example, IP addresses have a 32 bit address space [6], thus giving rise to the need to distribute dynamically the correspondences between a <PROTOCOLTYPE, PROTOCOL-ADDRESS> pair, and a 48 bit Ethernet address. 


\section{EXAMPLE ARP OPERATION}

Here is a review of the operation of ARP as defined in RFC-826 [5]. Let hosts $X$ and $Y$ exist on the same Ethernet cable. They have physical Ethernet addresses EA(X), and EA(Y), and DoD Internet addresses IPA $(X)$, and IPA $(Y)$. Let the Ethernet type of Internet be $\mathrm{ET}(\mathrm{IP})$. Host $\mathrm{X}$ begins an application, and sooner or later wishes to communicate an Internet packet to host $Y$. Host $X$ has knowledge of the Internet address of $Y$, i.e., (IPA(Y)), and informs the lower level that it wishes to talk to IPA(Y). The lower-level subsequently consults the ARP Module (ARM) to convert $\langle E T(I P), \operatorname{IPA}(Y)>$ into a 48 bit Ethernet address but because $X$ has not talked to $Y$ previously, it does not have this information in its Translation Cache (TC). It discards (or queues) the Internet packet, and creates a new Address Resolution packet with:

PACKET FIELD

HRDTYP

PROTYP

HRDLEN

PROTLEN

ARPOPC

SOURCE HWR

SOURCE PROT

TARGET HWR

TARGET PROT
VALUE ASSIGNED

ETHERNET

ET (IP)

length (EA (X))

length (IPA (X))

REQUEST

$E A(X)$

$\operatorname{IPA}(\mathrm{X})$

don't know

$\operatorname{IPA}(\mathrm{Y})$

It then broadcasts this packet to all hosts on the connecting cable. Host $Y$ picks up this packet and determines that it understands the hardware type (Ethernet), that it speaks the indicated protocol (Internet), and that the packet is for it, that is, TARGET PROTOCOL ADDRESS = IPA $(Y)$. Replacing any previous entry, it enters the information that $\angle E T(I P)$, IPA(X) translates to EA(X). It then learns that this is an ARREQ packet, so it swaps fields, placing EA(Y) in the new sender Ethernet address field SOURCE HARDWARE ADDRESS, EA(X) as TARGET HARDWARE ADDRESS, IPA(X) as TARGET PROTOCOL ADDRESS, IPA(Y) as SOURCE PROTOCOL ADDRESS, and sets the opcode to REPLY. The packet is then sent with direct routing address information to EA(X). Thus, $Y$ now knows how to send to $X$, but $X$ still doesn't know EA(Y). 
When $X$ receives the ARREP packet from $Y$, it gets the address information into its translation cache ET(IP), IPA(Y)>-->EA(Y), notices that it is a REPLY, and discards the packet (i.e., disposes of the dynamic packet buffer). However, if the original Internet Module packet had been queued, it could have been accessed and given the full addressing information from the translation cache. Alternatively, had it been discarded, the higher level would have succeeded on a subsequent attempt, and the Internet packet would be transmitted immediately.

\section{OBTAINING GREATER NETWORKING RANGE}

There are many benefits to be gained in dividing a large multiuser network into smaller, more manageable networks. These include : Data Security; Overall Network Reliability; Performance Enhancement; not to mention the most obvious: Greater Networking Range. In some network technologies, cable length may be stipulated not to exceed a certain range due to electrical limitations. By installing a Bridge, this restriction is effectively eliminated. An important consideration is the effect the induced Bridge delays will have on the protocol timeouts in operation on each LAN/Subnet. Careful analysis of upper bounds on timeouts would have to be made in order to gain full benefit from the increased range. In the case of Ethernet the following system parameters exist [11], [12]:

- the bus bandwidth is 10Mbit/s

- the maximum node-to-node cable length is $1500 \mathrm{~m}$

- the maximum point-to-point link cable length is $1000 \mathrm{~m}$

- the maximum number of repeaters between two nodes is two

- the worst case end-to-end bus propagation delay is 22.5 us

- the jam time after collision is 32bit

- the minimum interframe time is 9.6 us

- the slot size is 512 bit $=51.2$ us

Once a decision has being taken to subnet, the resulting subLANs may be connected by including a Bridge to link them together and providing a protocol which makes the collection of subnets appear as a single network. The basic idea of the Bridge providing 'repeater' facilities would not suffice in this application. Moreover, the Bridge would have to have further 'intelligence' to enable it to select those packets which are destined for remote networks based on 
the protocol address of the target host. Thereby preventing it from forwarding packets needlessly that will not be accepted. If this procedure was not adhered to, the channel bandwidth on the remote networks would be inundated with packets, causing local valid traffic to backoff and the efficiency of the respective networks to rapidly decrease.

One problem fundamental to the operation of the Bridge is how it discovers on which LAN a particular host is interfaced. If there are only two LANs in the system, each will have a dedicated cache at the Bridge, and when a packet is received at the particular interface, the source host's address parameters are entered in the respective LAN cache. However, when we consider a Multi-LAN environment, the procedure becomes more complicated.

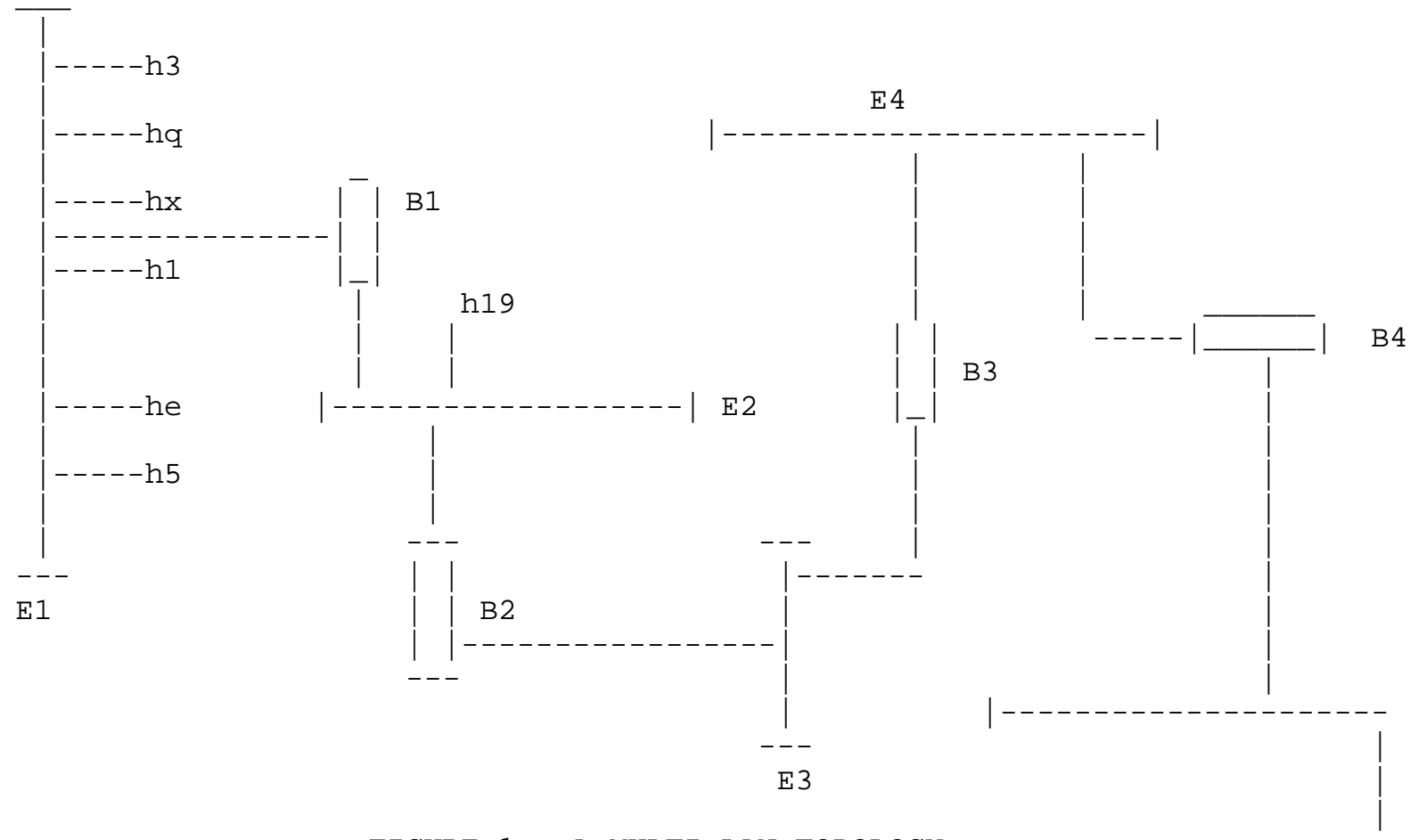

FIGURE 1. A MULTI-LAN TOPOLOGY

In the normal set-up, whenever B3 or B4 would receive a packet on E4, they would both update the caches on their E4 interface. In addition, a method must be provided to permit B4 to distinguish between packets arriving on E4 from E1, E2, E3, and those which actually originated on E4. 
This is so that packets can be categorized as being of remote or local source and processed accordingly. The most obvious solution is for each Bridge to act as an AGENT and plug in its address as the source of any packets it cascades to a remote network, instead of the packet being cascaded with its original source address. At Bridge boot, it may issue a broadcast request for all locally connected hosts/devices to return their local network protocol addresses. On subsequent receipt of this information, the Bridge could then update the cache for each of its interfaces so that it would now have a base from which to perform future operations.

The alternative to this automatic procedure is to permit manual intervention in the Bridge software which could be activated by the network manager in order to key in the addresses of the hosts connected to each LAN interface.

Thus, having provided a means for the Bridge to obtain the original state of the LAN addresses when it boots, how then does the Bridge distinguish the arrival of a new host on the locally connected system from transmissions which were sent from a remote source and cascaded by an adjacent Bridge? Two approaches are currently under consideration to solve this problem, namely Explicit subnets, and Transparent Subnets [4], [7], [9], [14].

In the Explicit Subnet approach, the location of the host in the system is important. The address of the host in the protocol suite will reflect which subnet the host is interfaced to. Consequently the protocol address space is divided into a three level hierarchy of <network, subnet, host>. Within the Internet there are five addressing divisions in operation [10], classes A, B, C, D, and E. Classes D and E relate to an addressing technique that will be used for management of multi-casting groups and will not be discussed here. With such a structure, it is possible to provide an address mask at each interface so that received packets may have their source address fields examined and compared with the address mask of this LAN. In so doing, the component which is being verified is actually the subnet address. If the masking operation is successful the source must exist on this LAN, otherwise it must be remote.

With the Transparent scheme, the first time a newly booted host 'speaks' it will be looking for addressing information (probably using BOOTSTRAP [1], RARP [2] or ARP [5]). Accordingly, the Bridge will detect these respective requests and be in a position to perform operations on the address parameters. The current approach in Transparent Subnetting is that before any such requests can be cascaded by the Bridge to an adjacent LAN, that Bridge will place its interface address parameters into the source address fields, thus acting as the AGENT. Therefore, this Bridge will 'see' either 
packets arriving from the remote Bridge address, or local packets. By virtue of the RARP/ARP operation, which hosts perform when they first come up, any hi-level packets received on to the network not having the bridge address, and not having a mapping in the cache for that LAN, can be considered as being remote.

Currently, there is a move toward the Transparent subnet proposal originally described by postel [7]. This has been due mainly to practical problems of incompatible implementations from different vendors, and the restrictions that the Explicit address space place on the adaptability of the system to change (class C addresses are not flexible enough for the Explicit scheme). It is also the opinion of the Author of this paper that the Agent technique adopted by the Bridges could have shortcomings in a dynamic environment which would be detrimental to its operation; for example, where the bridges themselves relocate or crash, or in the management of the "Agent For Who" cache at the bridge. Insofar as Loop Resolution and SelfStabilization after failure are Bridge problems that need to be addressed, it is strongly felt their satisfactory solution will be supported by elimination of the Agent technique [13].

\section{BRIDGE OPERATIONS}

Referring to figure 1, assume that at some stage during its processing [E1H3] wishes to communicate with [E2H19]. [E1H3] obtains knowledge of the Internet address of [E2H19] from its translation cache, but will not require the knowledge that [E2H19] exists on a completely different subnet. [E1H3] calls its Internet Module to transmit the packet. As detailed, the usual procedure of passing control to its ARM is performed in an attempt to obtain a translation. If we assume that [E1H3], and [E2H19] have not talked before, the ARM in [E1H3] will not be able to resolve the addresses on the first attempt.

In such a case, an ARREQ packet is assembled and broadcast to all hosts on the network [E1]. The packet traverses the cable and is eventually picked up by the (B1) Bridge Address Resolution Module (BARM), whereupon it determines whether or not it should intervene in the request. If the target is determined as remote (i.e., having no match in the local cache), the BARM examines its Global Translation Cache (GTC) to determine if it has an entry for <protocol, [E2H19]>. Should a mapping be obtained at the Bridge, there is no need for the broadcast REQUEST packet to be cascaded on to the remote network [E2]. It is therefore assumed that the entries in the GTC reflect the most current addressing information. A match thus obtained, the original ARREQ packet buffer is adapted as required and returned directly to [E1H3] via the Bridges hardware interface IFE1. 
On the other hand, should the Bridges' GTC have no information on [E2H19], the BARM would have to perform the following steps:

1. drop the current ARREQ from [E1H3],

2. create its own ARREQ using the Bridge source addresses and copy the target_internet_addr from the original [E1H3] ARREQ packet,

3. broadcast the ARREQ on network E2 via network interface IFE2, and go into a timeout awaiting a REPLY.

Should this timeout period expire, a number of retries will be permitted under control of the BARM. Alternatively, if a REPLY is received within the timeout interval, then the BARM will update its GTC. The ARM of [E1H3] next will attempt to transmit another ARREQ, but this time a mapping will be obtained at the BARM'S GTC, and the appropriate REPLY will be returned.

Part 1 has described the state of the art of the behaviour of Address Resolution. Part 2 now extends the study to the more serious problem of rebooting hosts in a multi-LAN system of Ethernets, and the effects such changes have on the integrity of state information held in ARP caches and routing tables.

PART 2

THE CAPTURE OF REBOOTS

Because Address Resolution packets are broadcast, all hosts on the connecting cable including the Transparent Bridge will pick them up and determine what they are. Referring to figure 1, it may well be the case that a host on E1 wishes to communicate with a fellow host on the same physical ether. Hence, if $\mathrm{Hx}$ wishes to talk to Hw on the same ether, but has not done so previously, it will broadcast an Address Resolution packet in the normal fashion. The Bridge will also' 'see' the packet as it passes by, and will act as described above, unless that is, there is some method of preventing it doing so; there is no point in the Bridge invoking its ARM, and wasting processing time if the problem is going to be resolved locally.

It may occur however, that $\mathrm{H} 1$ wants to communicate with H5. If however, H5 has not talked with anyone before (i.e., it has been "dormant"), H1 will issue an ARREQ. The Bridge will not know that H5 is local because it won't have been entered in the local address cache from previous conversations. To avoid broadcasting an ARREQ to all networks/subnets, one way around this problem is to set up the contents of the local cache at Bridge startup time. Therefore, the 
Bridge will already know not to intervene. Thus, if the Bridge (with 2 nets) finds that a particular IP destination address is not in the local cache of interface 1, it would have to examine its GTC and scan it for a mapping. Should no mapping be obtained at interface 2 , one of two possibilities exist:

1. the target host doesn't exist locally

2. the caches are corrupt (the eventuality of this should be negligible!)

If it is assumed that each of the translation caches contains have the most recent addressing information regarding its own domain of the network then, in this example, if the Bridge does not get a mapping at the GTC it would appear that the host must exist remotely from E1, and E2.

Such a conclusion would ignore cases in which a host unplugs from a particular hardware interface and plugs into another hardware interface, or where logical names are reassigned to different interfaces due to host user change. Either of these events could happen had the host being accessed on E2, which would mean that a REBOOT has taken place.

Anticipating these possiblities local caches are essential. In normal operation, the Bridge will process and forward IP packets received from one network, and destined for another. If the Bridge picks up an ARREQ, it will first look for a mapping in its GTC before discarding the original $A R R E Q$, and transmitting its own to the remote network. In any case, the Bridge will always examine the local cache entries at the receiving interface, so that it may determine if the target address is local or remote. When the Bridge first scans the local cache, it does so with the source IP address as the key. If no mapping is retrieved, it then scans the GTC with the same key.

Should a mapping now be obtained, it remains for the Bridge to insert the source IP into the local cache, where it has either been previously deleted or corrupted.

However, if the source IP exists in the respective local cache, the validity of the source Ethernet address should also be verified by examining the respective entry in the GTC. A scan of the GTC is then performed with <protocol, source_prot_addr> as the key. If a mapping is retrieved, the respective <et_addr> should be checked against the source Ethernet address in the packet header. If the addresses do not match, then we have uncovered a Hardware Reboot condition (i.e., a change in Ethernet ID). On the other hand, should the scan of the GTC with <protocol, source_prot_addr> fail to obtain a mapping, then the Bridge would scan the GTC with the current Ethernet address in 
the packet header. If this obtains a mapping, then a Protocol Reboot condition (i.e., change in logical ID) has been detected.

In the next section, the implications of these forms of 'Reboot' are discussed.

\section{REBOOT SCENARIO}

In normal operation, packets will uneventfully traverse each subnet either as complete Internet packets, broadcast ARREQ's, or direct ARREP's. The Bridge attached to each subnet will ' hear', and 'see' all packets as they travel past its connected interfaces. Because of the existence of the local caches at each interface, the Bridge can decide whether or not to intervene. In general circumstances, each host on the Catenet will have a translation cache containing <protocol, source_prot_addr, source_et_addr> entries for all packets it has observed. Most of these entries will have been due to processing ARREQ packets, which were broadcast, and by receiving REPLY packets. In accordance with the foregoing, the Bridge will have a cache attached to each subnet interface containing entries for protocol addresses.

Within the Bridge's Global Translation Cache (GTC) will be entries of all <protocol, source_prot_addr, source_hrd_addr> triplets relating to valid hosts which have been recognised. If we assume that we have just connected up a Catenet such as that illustrated in figure 1 , then at power-up no stations will have knowledge about their neighbours. If the Bridges are to remain transparent, the translation caches at each host will be totally empty. The only addressing details that will be in existence will be the protocol addresses stored in the local caches of the Bridges.

The hosts subsequently begin to run applications and will want to communicate with one another. The first ARREQ is broadcast on the respective subnet and all hosts, including the Bridge's interface to the subnet, will pick it up and store the details. If, for example, $\mathrm{Hx}$ issues an ARREQ for $\mathrm{Hq}$, the Bridge will not intervene since there is no need (providing no reboot has occurred at $\mathrm{Hq}$ ). However, if $\mathrm{Hx}$ wishes to talk with $\mathrm{Hz}$, Bl will determine that the target IP in the respective ARREQ does not exist in the local cache of IFE1, so it will examine the GTC, with the <protocol,target_prot_addr> of $\mathrm{Hw}$ as the key.

It is assumed that there will be a timeout mechanism in operation at the source of any packet. In addition, the Bridge may also place the target address in a 'search list' of currently sought hosts, so as to prevent ARREQs from different sources being cascaded for the same target. Under these conditions, $\mathrm{Hx}$ may re-issue its original ARREQ, 
but will be ignored until the host Hw has replied to the ARREQ transmitted by the Bridge.

NORMAL RUNNING STATE

Assuming that a few ARP's have been issued, IP packets will start traversing the Catenet with full addressing information. Again, the Bridges will 'see' all the packets. If we extend the situation one step further, and assume that several conversations have taken place across the Catenet, there will be entries in the translation caches of the hosts concerned, regarding the <protocol, target_prot_addr,target_hrd_addr> triplets of those hosts with which the conversations took place. The Bridges also, will have details in their GTC's for packets which they cascaded.

If a host is relocated, any connections initiated by that host will still work, provided that its own translation cache is cleared when it does physically move. However, any connections subsequently initiated to it by other hosts on the Catenet will have no particular reason to know to discard their old translation for that host. Ideally, 48 bit Ethernet addresses will be unique and fixed for all time.

\section{RECOGNITION OF THESE REBOOT CONDITIONS}

With reference to figure 1, assume that for some reason a fault occurs on the hardware interface of $\langle E 1 H e\rangle$. The result of this is that a new interface is installed with a newly acquired hardware address. When $\langle\mathrm{E} 1 \mathrm{He}\rangle$ is powered up, the previous contents of its translation cache are cleared and it has no recollection of local, or remote host addresses. Accordingly, <ElHe> begins to issue ARREQ'S to hosts it requires. Whenever $\langle\mathrm{E} 1 \mathrm{He}>$ transmits its first $A R R E Q$, it could be termed a 'HELLO PACKET', since everyone on the subnet can pick up the packet, and store the relevant information in their translation caches. Within hosts, a mapping will be found on the old <protocol, source_prot_addr> pair, and the current <et_addr> of the packet header will replace whatever is entered in the translation cache.

At this point it would be easy for each host with an entry to recognise the Hardware Reboot situation and inform the subnet with a respective broadcast reboot packet. But allowing such a procedure would be extremly inefficient on the broadcast medium, and would drastically outweigh any improvements in performance which might be obtained in the long term. In any case, given the fact that the ARREQ is broadcast, all stations on the subnet will recognise the reboot. The important point to consider is the effect such a reboot will have on subsequent conversations which are initiated remotely. 
Can redundant transmissions be thwarted before they tie up processing time on hosts en-route to the rebooted target? How these difficulties are resolved is critical to the level of performance obtained in a Catenet configuration. Since it is not optimal for hosts to inform the system of a reboot, it is left to the Bridge. Whenever the Bridge receives a packet, be it IP, or ARP, it examines the source address parameters in the packet header, in the hope of detecting any incompatibilities between them and the entries in its caches. There are three distinct possibilities, namely, a difference in the 48 bit hardware address only, a difference in the protocol address, and two completely new addresses. If an incompatibility is discovered, a "REBOOT" packet is constructed and issued on all remote interfaces containing the appropiate information, allowing Bridges to update their GTC's and generic hosts their ARP caches.

The structure of the Reboot packet is as depicted in figure 2 .

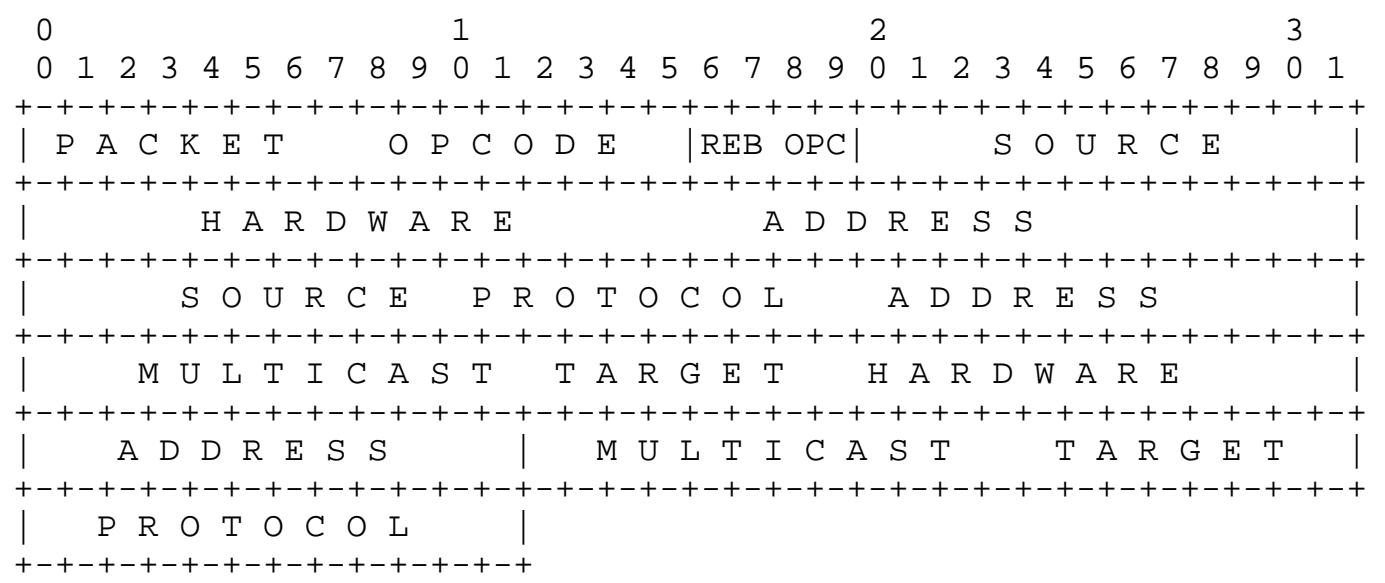

\section{$------->$ NEXT FOLLOWS A VARIANT FIELD ON REBOOT OPCODE}

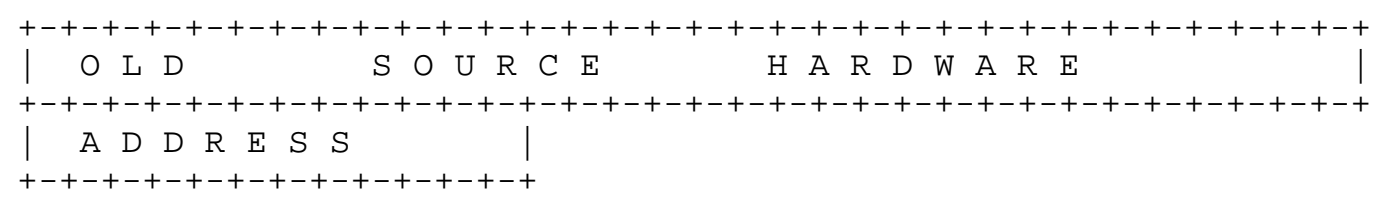

OR

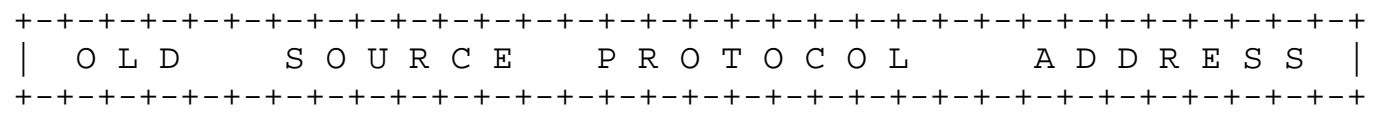

FIGURE 2. REBOOT PACKET 
The following definitions apply:

$\begin{array}{ll}\text { PACKET FIELD } & \text { VALUE } \\ \text { OPCODE } & \text { REBOOT } \\ \text { REBOOT OPCODE } & \text { HARDWARE } \\ \text { REBOOT OPCODE } & \text { PROTOCOL }\end{array}$

The format is then as follows:

48 bit broadcast Ethernet address for the destination,

48 bit Ethernet address of source Bridge,

16 bit Protocol type = PACKET OPCODE - REBOOT .

\begin{tabular}{|c|c|c|}
\hline FIELD & FIELD SIZE & DESCRIPTION \\
\hline HRDLEN & 4 bit & byte length of Ethernet address \\
\hline PROTLEN & 4 bit & byte length of Protocol address \\
\hline \multicolumn{3}{|l|}{ SOURCE } \\
\hline ADDRESS & 32 bit & current protocol address of host \\
\hline \multicolumn{3}{|l|}{ TARGET } \\
\hline ADDRESS & 32 bit & broadcast target protocol address \\
\hline \multicolumn{3}{|l|}{ REBOOT } \\
\hline OPCODE & 4 bit & will be either PROTOCOL or HARDWARE \\
\hline if $\quad$ PROTOCOL & 32 bit & old protocol address \\
\hline else HARDWARE & 48 bit & old hardware address \\
\hline
\end{tabular}


As shown, depending on the REBOOT-OPCODE, the structure will continue with either the 48 bit old hardware address or the 32 bit old protocol address. The choice of a variant packet structure is for reasons of curtailing the size of the packet to the fields that are truely necessary in each situation. From this Reboot packet structure, the process of generating such a packet can be considered. When the Bridge algorithm detects a reboot, it should create a reboot packet structure containing the relevant addressing information and subsequently multicast it on the interface(s) which access(es) the remote subnet(s). The decision as to which interface(s) is/are local, and which is/are remote, can be resolved automatically whenever a packet is received. With respect to this packet transfer the receive interface at the Bridge becomes local, and all others are tagged as remote.

Thus, hosts on the subnet remote from the reboot are informed of the situation immediately as it is detected by the Bridge. In the Catenet configuration illustrated in fig 1, this will have the effect of updating the Translation Cache within each host, whenever it receives the packet. If for example, <E4HW> reboots under hardware, B3 will detect this occurance. There is no reason for the subnets E1, E2, E3 to be aware of this episode. In normal operation, B3 will recognise the reboot from the first $A R R E Q$ issued from $\langle E 4 \mathrm{HW}\rangle$. With this reboot detection facility, B3 will be in a position to inform the hosts on E1, E2, and E3. B3 can then create and issue the Reboot packet via its interface with E3. When B3 picks it up, it will update its own caches and subsequently cascade the packet onto E2, where it will be passed on to El via B1.

\section{ARGUMENTS FOR REBOOT PACKETS}

It is envisaged that introducing Reboot packets, will serve to enhance the bandwidth achievable within a Catenet system. Problems of addressing 'dead' hosts will no longer exist in a correctly functioning configuration. Translation Caches will have on hand the most recent addressing information available, which should also serve to enhance the performance of the routing strategy in operation. Multiple, redundant processing of packets destined for 'dead' hosts will be avoided. Weighing this against the processing involved with a single multicast of Reboot packets, it is expected that the latter will be is the most economically viable in relation to the long-term traffic presented to the system.

\section{CONCLUSION}

It appears that reboots are becoming increasingly common on internet networks. Many sites use Personal Computers (PC) as terminals and the typical way to finish a session is to switch them off! with the 
increasing popularity of multitasking Operating systems on these types of machines, problems are more likely to occur, particularly when the PCs are diskless, or participating in a distributed file system of some kind. Given the importance of correct addressing in communications networks running Ethernet, it is anticipated the reboot mechanism described will serve to improve the correctness and validity of the protocol/network address mappings which may be stored in the translation caches. To this degree, simulation is expected to show that the volume of invalid traffic will decrease, to the benefit of hosts, Bridges and servers alike. Likewise, ratification of the routing policy is anticipated and since redundant/obsolete packets will be thwarted, the efficient utilization of available channel bandwidth across the catenet is also expected to improve. Thus, effectively increasing Catenet throughput for 'valid' packets, and therefore enhancing the level of service provided to the end users.

It is obvious that the proposed scheme implies the alteration of the packet processing code in Bridges/Gateways. The point to remember is the increased favour with which larger, more complex Multi-LAN systems of Ethernets are being received. The recent adaption of extra telephone cables to serve as the transmission media for the Ethernet can only result in installation costs being reduced, therein making the Ethernet more attractive within large corporate buildings, etc. It is sensible to suggest that the probability of host address re-assignment shall increase in proportion to the number of physical systems attached, component failure rate (for whatever reason), relocation of resources, and the size and turnover of the workforce (i.e., people moving from one room to another). Simulation experiments are currently being developed to analyse the resultant traffic patterns under this scheme, and it is hoped to highlight thresholds where adoption of the scheme becomes a necessity.

In addition, the Author is currently extending the boundaries of this problem to encompass the reboot, or relocation of Bridges themselves. Involved with this are the phenomena of loop resolution, load sharing and duplicate packet suppression. It is envisaged that a selfStabilizationg Bridge Protocol will result that will be more "lightweight" than those adhering to the spanning Tree Algorithm.

The Author would appreciate feedback/comments on this RFC. My network address is: CBAD13\%UCVAX. ULSTER. AC.UK@CUNYVM. CUNY. EDU.

\section{ACKNOWLEDGEMENTS}

The Author acknowledges with gratitute the help and comments contributed by Mr. Piotr Bielkowitz (Supervisor) of the Computing Science Department, and the time devoted my Mr. Raymond Robinson for painstakingly preparing the first draft of this paper on 'Pagemaker'. 
Thanks are due also to Dr. M. W. A. Smith of Information Systems for his assistance. Finally, this work was supported under a grant from the Department of Education for Northern Ireland of which the Author is extremely grateful.

\section{REFERENCES}

[1] Croft, Bill, and John Gilmore, "Bootstrap Protocol", RFC-951, Stanford University, September 1985.

[2] Finlayson, Mann, Mogul, and Theimer, "A Reverse Address Resolution Protocol", RFC-903, Computer Science Dept, Stanford University, June 1984 .

[3] Lorimer, Alan, and Jim Reid, "ARP Information Communique", Computer Science Dept, Strathclyde University, 1987.

[4] Mogul, Jeffrey, "Internet Subnets", RFC-917, Computer Science Dept, Stanford University, October 1984.

[5] Plummer, David, "An Ethernet Address Resolution Protocol", RFC826, MIT, November 1982 .

[6] Postel, Jon, "DARPA Internet Program Protocol specification", RFC-791, USC/Information Sciences Institute, September 1981.

[7] Postel, Jon, "Multi-LAN Address Resolution", RFC-925, USC/Information Sciences Institute, October 1984.

[8] Postel, Jon, Carl Sunshine, and Danny Cohen, "The ARPA Internet Protocol", Computer Networks, no. 5, pp. 261-271, 1981.

[9] Postel, Jon, and Jeff Mogul, "Internet Standard Subnetting Procedure", RFC-950, USC/Information Sciences Institute and Stanford University, August 1985.

[10] Reynolds, Joyce, and Jon Postel, "Assigned Numbers", RFC-1010, USC/Information Sciences Institute, May 1987.

[11] "The Ethernet: a local area network, data link layer and physical layer specification", Version 1.0 DEC, Intel and Xerox Corporations, USA 30 september 1980).

[12] Hughes, H.D., and L. Li, "Simulation model of an Ethernet", Computer Performance, Vol 3, no. 4, December 1982.

[13] Parr, Gerald P., "Address Resolution For An Intelligent Filtering Bridge Running On A Subnetted Ethernet System", ACM 
SIGCOMM Computer Communication Review, (July/August 1987), vol. 17 , no. 3 .

[14] Smoot, Carl-Mitchell, and John S. Quarterman, "Using ARP to Implement Transparent Subnet Gateways", RFC-1027, Texas Internet Consulting, October 1987. 